\title{
Human Heterogeneity and Survival of the Species: How Did It Arise and Being Sustained?-The Conundrum Facing Researchers
}

\section{David A. Hart $1,2,34$}

${ }^{1}$ Department of Surgery and Faculty of Kinesiology, University of Calgary, Calgary, Canada; ${ }^{2}$ Bone \& Joint Health Strategic Clinical Networks, Alberta Health Services, Edmonton, Canada; ${ }^{3}$ McCaig Institute for Bone \& Joint Health, University of Calgary, Calgary, Canada; ${ }^{4}$ Centre for Hip Health and Mobility and Department of Family Practice, University of British Columbia, Vancouver, Canada

Correspondence to: David A. Hart, hartd@ucalgary.ca

Keywords: Human Heterogeneity, Species Survival, Genetic Variation, Evolutionarily Conserved, Pandemics and Survival

Received: April 2, $2021 \quad$ Accepted: May 11, $2021 \quad$ Published: May 14, 2021

Copyright $\odot 2021$ by author(s) and Scientific Research Publishing Inc.

This work is licensed under the Creative Commons Attribution International License (CC BY 4.0).

http://creativecommons.org/licenses/by/4.0/

\section{(c) (i) Open Access}

\section{ABSTRACT}

Current humans, Homo sapiens, are genetically and epigenetically very heterogeneous, and subsequently also biologically and physiologically heterogeneous. Much of this heterogeneity likely arose during evolutionary processes, via various iterations of humanoid lineages, and interbreeding. While advantageous from a species perspective, the heterogeneity of humans poses serious challenges to researchers attempting to understand complex disease processes. While the use of inbred preclinical models makes the research effort more effective at some levels, the findings are often not translatable to the more heterogeneous human populations. This conundrum leads to considerable research activity with inbred preclinical models, but modest progress in understanding many complex human conditions and diseases. This article discusses several of the issues around human heterogeneity and the need to change some directions in preclinical model research. Using newer Artificial Intelligence and Machine Learning approaches can begin to deduce important elements from the complexity of human heterogeneity.

\section{INTRODUCTION}

When a pandemic such as the current viral COVID-19 version arises, some infected people have a severe disease course, with deaths and prolonged hospitalizations. In contrast, others have a very mild dis- 
ease course with almost no symptoms. This is not a unique scenario as it played out similarly with the Spanish flu pandemic in 1919-1920. With the AIDS virus, the outcome is more uniform with regard to advancement of the viral infection proceeding to a high proportion of deaths until drug interventions became effective. However, a small percent of infected people, such as some infected prostitutes in Nairobi, Kenya remain infected but the disease does not progress to full blown AIDS due to their genetic make-up. This pattern of response to a microorganism is not new as it played out similarly during the plagues of Europe mediated by other vectors and bacteria such as Yersinia pestis. Again, not every individual became overtly infected and not all infected individuals died, but $>50 \%$ did die of the plague in those pre-antibiotic ages [1].

In addition, there is some evidence that certain blood group variations may offer some protection against parasites such as malaria, so this variation in populations is not restricted to specific types of micro-organisms. Interestingly, with more people refusing to become vaccinated, there have been more cases of what used to be common diseases such as the measles, and only a small percentage of infected people actually die of the disease. Similarly, recent data indicate that people with certain blood group antigens may have more risk for severe disease with COVID-19 than others, but this biomarker is not consistent (discussed in [2]). Thus, in human populations it may be advantageous for the survival of the species to have genetic variation, particularly if maintained in the hetero-zygotic state. This raises the possibility that what may be advantageous in the hetero-zygotic state could be detrimental when homozygous for the same variation, such as with some lipid storage diseases and others such as the cystic fibrosis gene(s).

There are two important points to be derived from such response patterns. The first is that Home sapiens appear to thrive on genetic heterogeneity as it ensures survival of the species to a number of insults. The second is that chronic exposure to a potential pathogen would lead to a discernable shift in the gene pool of an isolated population that did not interbreed with other populations. Current humans apparently did inter-breed with Neanderthals and other now extinct populations (i.e. based on DNA sequencing) (discussed in $[3,4])$. A recent report has indicated that some of this inherited DNA may play a role in COVID-19 infections [5].

While such mechanisms such as those discussed above are advantageous for some populations, the converse can be detrimental. That is, isolated populations can be selected over time to be resistant to the endogenous pathogens or potential insults that exhibit risk for survival but have little resistance to exogenous pathogens. Examples include survivalist populations in North America, Hawaii, or Australia who were exposed to microorganisms carried by explorers from Europe or other indigenous traders, but had little to no resistance based on their isolation for thousands of years, and died in large numbers, nearly wiping out several cultures. Therefore, such populations likely evolved to be somewhat resistant to local endogenous organisms, but not those from elsewhere. It is of interest that the reverse effect-that of local indigenous cultures infecting explorers and traders from Europe is not discussed, but if it did not happen it is curious that indigenous populations did not harbor a set of endogenous pathogens that were reflected by their isolation for millennia.

Likely, it is not just microorganisms and pathogens that have driven humans to be very heterogeneous. Other factors such as food insecurity and ability to sequester energy in the form of fat to survive in times of scarcity would also be advantageous. Currently, there are over 100 genes that have been implicated in the current obesity epidemic (discussed in [6]), likely genes that date back into history for their time of appearance and subsequent selection. Similarly, in many places on the planet salt was in scarce supply and thus, an ability to retain salt, a critical commodity for maintaining physiologic function via the kidneys or sweating would be advantageous for survival. However, such an ability could be detrimental to kidney function if salt became plentiful and was over consumed (such as in many Western societies).

Thus, isolation can have both positive and negative influences on human heterogeneity, and chance encounters can have severe implications for any specific gene pool. However, more often, the process for selection occurs over many generations as those with the advantageous trait would be selected for from breeding success. 


\section{HOW COULD SUCH HUMAN HETEROGENEITY ARISE AND BE MAINTAINED?}

As the lineage for Homo sapiens likely tracks back millions of years, the genomes of current humans contain many of their $\sim 20,000$ genes and associated millions of SNP and variants in both coding and non-coding elements that are both essential for life, essential for being human, and those that have contributed to survival over the millennia. There is also the potential for genetic exchange between humans and other human lineages such as the Neanderthals and other human lineages that have died out. Based on DNA comparisons, many Homo sapiens have variable contributions of Neanderthal DNA and others such as the Denisovans $[3,5]$.

For some genes, heterogeneity can be maintained at the level of successful reproduction. For example, genes in the major histocompatibility complex (MHC) are quite complex (MHC Class I, II and III, plus many minor loci) and may be the source for risk for a number of diseases (discussed in [7-9]). Based on studies from the 1970s and 1980s [10-12], human breeding pairs with very similar MHC antigens often have trouble carrying a fetus to term. This likely resides in the fact that the fetus is an allograft to the mother, and histocompatibility leads to better implantation and placental development [12]. Thus, the heterogeneity of the sperm donor would be selected for regarding a successful reproductive event with the recipient. In addition, it is likely that much of the advantageous aspects of the heterogeneity may reside in heterozygosity, with homozygosity leading to either a defective offspring or one with a disease that limits survival (i.e. cystic fibrosis, Tay-Sachs disease, Huntington's chorea).

Thus, heterogeneity likely arose during the multiple iterations of humans that preceded Homo sapiens, during the successful migration patterns out of what is believed to be Africa, and eventual domination of nearly all of the continents, interbreeding with many groups, surviving the frequent threats from cyclic climate alterations, from efforts to maintain food security, and to resist microbial exposures. It is also maintained by both the basics of reproduction, and the drive to maintain heterozygosity.

Humans, like pigs and most other animals appear to avoid inbreeding to reduce the onset of homozygosity. Thus, the ability to generate inbred mice is likely a mammalian anomaly, and as such its usefulness to model humans is an issue to be reconciled. Certainly, zoos and related enterprises are quite aware of such issues regarding genetic heterogeneity when it comes to rescuing species from the brink of extinction.

While not normally thought of as having an impact on gene pools and human heterogeneity, the net effect of modern medicine, the use of antibiotics, public health measures, and drugs certainly has impacted the survival of many humans who would have died previously during evolution, at least up to the last 100 300 hundred years. Thus, while human lives have been saved from the above interventions to mitigate risk for death, the net effect may be to enhance heterogeneity by protecting susceptible individuals, but whether this has been a good thing for the species remains to be determined in the long term.

Medical science has also elucidated some human heterogeneity that was previously either unknown or under appreciated. Some drugs such as those that are delivered as a prodrug that needs to be activated in the liver by a P450 enzyme can be lethal for those lacking or deficient in a specific isoform of the P450 family $[13,14]$. This deficiency is apparently silent in most humans unless challenged, so likely arose through some mutational event and has been maintained. By screening for the deficiency, those at risk for adverse reactions to the drug can be avoided, and the efficacious delivery of the prodrug to others can exert benefit. Additional heterogeneity in relation to drugs is associated with sex and race (related to effective dosing) (discussed in [15-17]), and other genetic variations [13, 14]. All of these factors point to the need for precision medicine approaches to address this heterogeneity.

Human heterogeneity has also been revealed by space flight, a condition that could not have been anticipated by evolution on Earth (unless we are missing something from past history), but heterogeneity that has certainly been shaped by the boundary conditions of Earth. Therefore, going into space has revealed considerable heterogeneity in the physiological responses of individuals to microgravity. Thus, there is wide variation in bone loss $(0.1 \%-2 \%$ per month), cardiovascular changes, alterations to vision, and others (discussed in $[18,19])$. Therefore, when individuals as relatively young healthy people go into 
space, these otherwise silent variants while on Earth, become evident. However, they may not be silent during aging and could contribute to previously unconnected links to disease [20,21]. Such genes and their variants may be part of the "use it or lose it" paradigm of life in a $1 \mathrm{~g}$ environment, a paradigm whose integrity may be compromised during the process of aging.

Female Homo sapiens are also heterogeneous with respect to one of their life transitions, namely menopause. Some females develop osteoporosis (with wide variation in the rate of bone loss), obesity (with unique fat distribution compared to males), osteoarthritis of the knee and hip (rates of OA in females after menopause $>2$ - 5 times that of males), cardiovascular event risk (appear to be protected prior to menopause but not after), dementia ( $73 \%$ of dementia patients are female after menopause), and sarcopenia (evidence not strong regarding sex differences). Not all females develop all of these conditions and thus, there are isolated subsets that have risk. Some of this risk is apparently genetic (i.e. development of osteoporosis in families). Why such disease risk is maintained is not clear, but the risk after menopause may offer reproductive advantage in earlier life (i.e. calcium mobilization from bone during pregnancy and lactation and then ability to replace post-lactation [22]).

Therefore, Homo sapiens are very heterogeneous from the genetic and physiological perspective, and benefit is likely derived from having such variation at a species level, but possibly not on an individual level. The level of complexity in the variation complicates attempts to understand the contributions of individual variants as likely some are major and others minor, and there is also likely redundancy regarding the functional contributions of several variants making elucidation of individual contributions to function even more difficult to unravel.

\section{DID HUMAN GENETIC HETEROGENEITY ARISE FROM PASSIVE OR ACTIVE PROCESSES, OR BOTH?}

If one assumes life arose on Earth, and the molecule chosen to use for continuity of that life from generation to generation, DNA was chosen in part due to its stability. However, the Earth uniquely has a background level of radiation that is variable based on location, and humans are sensitive to radiation, leading to DNA changes that could be silent, advantageous, or detrimental (i.e. cancer causing). Thus, in this scenario, the process would be passive and dependent on external influences. While the human genome has 20,000 genes, a considerable amount of DNA is in non-coding regions (i.e. introns in genes, non-coding miRNA), regions that were considered "junk" DNA not that long ago. Thus, many changes/"mutations" could occur in such regions and not influence the gene complement unless in a critical regulatory position, and thus contribute to heterogeneity. Other variation could arise in coding regions and unless affecting function, could also be silent, particularly in the hetero-zygotic state. However, such changes may not remain silent and appear to exert influence during aging past the normal life span of most humans for much of evolution (i.e. influence of the Huntington's chorea gene which becomes evident in the homozygous state at $\sim 35$ yoa).

Heterogeneity can also arise from the active process of DNA replication and active combining of DNA elements through splice variation. In addition, in the immune system, the example of VDJ joining of genetic elements to yield a function variable region for antibodies is an additional mechanism which can introduce "wobble" in the VDJ joining events. However, the retention of silent mutations in either coding regions or non-coding regions of DNA could contribute to heterogeneity that is beneficial when faced with unanticipated challenges (i.e. space flight, changes to the environment, availability of food sources). Likely, some of the genetic heterogeneity of humans also arose due to passive approaches, but certainly, environmental stresses could have driven some of the processes which became more optimized over time (i.e. obesity-related genes in times of food insecurity).

The above discussion raises the important question "Is threat resistance due to a lack of susceptibility, or due to an overt response"? Fundamentally, this is difficult to address. Given the extent of heterogeneity within a population, both scenarios are possible. For instance, in parts of Africa where malaria is endemic, the appearance of the sickle cell trait or alterations to the Duffy blood group which appears to convey re- 
sistance to malaria in the hetero-zygotic state (discussed in [23-25]), may have been due to forced evolution to resist an endogenous threat. In contrast, resistance to an exogenous threat encountered by a migrating population may be selection due to existing resistance in the population associated with the timelines needed to enhance survival. Also in relation to migrating populations is the loss of skin pigmentation in relation to survival. This loss may have accompanied migration to latitudes that required better vitamin $\mathrm{D}$ metabolism, and thus, loss of pigmentation that could interfere with such metabolism (discussed in [26]). However, this may have had consequences for other areas such as regulation of inflammation [27] and it is known that humans of the Black race and black mice both respond to lower doses and differently to the metabolic modulator lithium chloride/carbonate (discussed in [28]). In addition, the loss of pigmentation could relate to the melanocortins and their receptors, and lead to development of more skin cancer risk if the affected individuals migrated back to their central African origins [29]. As skin pigment loss has a number of potential consequences, it may have arisen over time to be selected for in order to enhance survival of the species. Interestingly, the Inuit of the Arctic derived their vitamin D from food sources and while living in a climate with very low exposure to the sun for months on end, did not appear to select for complete loss of pigmentation.

While the above question cannot be answered in full, the past 100 years have provided a unique opportunity to study the effect of removing a number of environmental threats on genetic resistance gene frequency and development of variants. In some regards, this may be analogous to the study of survivors of indigenous cultures exposed to pathogens brought by explorers or traders. That is, for the past 100 years, the advent of antibiotics, vaccines, and improved public heath have removed many environmental threats that previously killed many susceptible individuals. Thus, those particularly susceptible to such pathogens are now protected, and thus over several generations, will change the distribution in the gene pool.

\section{THE ISSUE OF HETEROGENEITY VS HOMOGENEITY IN RESEARCH}

To better understand Homo sapiens, particularly from a medical perspective, researchers do controlled studies with humans, or turn to preclinical models to provide insights. Such efforts are multi-billion-dollar enterprises around the world. While some research embraces the heterogeneity of humans, much of the endeavor attempts to minimize the heterogeneity as it can be detrimental to obtaining significant results for an intervention or drug with humans, or some manipulation in preclinical models that the researchers hope will allow the findings to be translated to humans.

The ultimate models for eliminating heterogeneity are the use of inbred mice that are nearly genetically identical. Rodents, particularly mice appear to be uniquely able to be extensively inbred, so the resulting strains have had heterogeneity bred out of them. Thus, individual strains differ based on the starting stocks or some other variable and can provide alternate options for assessing the effect of interventions. Thus, while mice have had millions of years to stabilize their genetic make-up to yield a species, humans have used mouse inbreeding to both maintain the outcome (but it is still a mouse), and to also eliminate as much heterogeneity as possible.

Furthermore, through genetic manipulation one can create knock-out mice lacking a specific gene, knock-in mice with a specific added gene, or even "humanized" mice with some human genes on a mouse background (discussed in [30,31]). Fortunately, even partial inbreeding of humans has detrimental effects, likely due to the fact the being heterozygotes for many genes may be an advantage but becoming homozygotes for the same genes could become a detriment. This is also seen in domesticated animals that are used for food (i.e. pigs, sheep, cattle) or pets (i.e. dog breeds and cats). The advantage of such small, inbred populations for research, in addition to their lack of heterogeneity, is also based in part on cost to house such animals, the ability of having a control and experimental population for comparisons, and the short gestation time to expand the population. Studies performed over many years with a specific set of mice $(\mathrm{NZB} / \mathrm{W})$ that develop a murine form of lupus indicated that treatment with $\mathrm{LiCl}$ led to vast improvement in lifespan, weight gain and fat deposition, but not humoral immune dysfunction (reviewed in [28]). 
However, $\mathrm{LiCl}$ was only effective in females and not males, indicating a sexual dimorphism. Further studies of the responsiveness of the parental strains (New Zealand Black and New Zealand White mice) revealed that the NZB strain was much more sensitive to $\mathrm{LiCl}$ in their response pattern than was the NZW strain. Interestingly, mice of the NZB strain also responded to low doses of $\mathrm{LiCl}$, and it has been noted that individuals of the black race treated with $\mathrm{LiCO}_{3}$ for bipolar disease, respond to lower doses of $\mathrm{LiCO}_{3}$ than do patients of the white race. However, the biological effects of $\mathrm{LiCl}$ on NZB/W females are very different than the effects of $\mathrm{LiCO}_{3}$ on human patients so while interesting, the outcomes from mice cannot be extrapolated to humans directly. In spite of this limitation of the findings, the race-associations may implicate Melanocortin-4 in the responses in both species, but if so, the post-modifications appear to be species-specific! Sexual dimorphisms in response to lithium salts in humans have not been definitively reported.

While using inbred rodents such as mice is viewed as "good science" and evidence of sophistication in research, the low translation potential of findings from mice to humans is a concern. With literally billions of dollars being focused on studies in mice, one wonders if the results are of interest as biological phenomena, or their relevance to humans or human disease. Thus, in this circumstance, eliminating heterogeneity may yield results that are of scientific interest as an anomaly of nature but elimination of heterogeneity has greatly diminished the potential relevance of the findings to a very, very small segment of the human population. Thus, the advantages of using homogeneous animals are obvious from a scientific perspective, but the outcomes are likely highly irrelevant to the human condition in most cases. This is also evident from studies using multiple inbred strains of mice where the outcomes are strain-specific.

In contrast, using large animals such as pigs and sheep that have physiologies or anatomies similar to humans (discussed in $[32,33]$ ) is much more costly than using small rodents, the numbers per group are thus potentially smaller due to cost, the animals are still quadrupeds, and their heterogeneity is often as broad as humans which is viewed as a limitation for group comparisons. Work from our own laboratories $[34,35]$ has used female sheep as a very sensitive model of post-traumatic osteoarthritis (PTOA) development and for many of the above reasons, a shift to an $\mathrm{N}$ of 1 experimental design was required due to heterogeneity in response patterns. In addition, because of cost/animal, it is often difficult to obtain grant funding to explore large animal models as many reviewers are committed to rodent models and group comparison paradigms rather than the $\mathrm{N}$ of 1 design.

Therefore, progress in solving many problems relevant to human health and disease has likely been slowed due to commitment to preclinical models that have limitations in translation potential to the human condition and not embracing models that likely have more similarities to human heterogeneity, physiology and outcomes. The other factor that comes from such studies is that even though humans and mice may share many genes, it is the regulation of those genes that is critical, not just the genes that are translated into proteins.

\section{HUMAN EXPERIMENTATION AND COHORT STUDIES: STRENGTHS AND WEAKNESSES}

The study of humans must meet a number of criteria regarding ethics, privacy, age and sex requirements, race, socioeconomic status, all factors that attempt to define both control populations that are "normal", as well as a population at risk for a particular disease or condition.

For some fairly prevalent conditions such osteoarthritis of the hip and knee, conditions that impact $\sim 10 \%-12 \%$ of the adult population, convening a cohort of normal individuals who are condition-free at the time of enrollment and then following them longitudinally with a set of surveys, imaging modalities, diagnostic tests, functional tests can lead to characterizing subsets of humans before and during development of the condition. Such studies include the OA Initiative in the US, the Johnston County Cohort (US), the United Kingdom Twin Initiative, and several others. Similarly, the Framingham Study (USA) has also provided good insights into the epidemiology of cardiovascular disease. If constructed to be sufficiently large and inclusive, such studies can incorporate human heterogeneity and use it to their advantage.

In contrast to cohort-based studies, drug or treatment focused clinical trials can take on different constructs, but they should encompass the population to be targeted and be large enough to identify po- 
tential subpopulations that may experience side-effects or be non-responders. While Randomized Control Trials (RCTs) are viewed as the "gold standard" there can be some variation in the construct regarding the control $\operatorname{arm}(\mathrm{s})$. That is, whether effectiveness of the drug/treatment in question is compared to standard of care vs placebo. For drugs affecting symptoms such as pain, the placebo effect can be quite significant and thus comparison to the standard of care should account for that potential confounder.

To generate a population for a clinical trial one could construct a large population with no constraints, or establish inclusion and exclusion criteria to restrict the heterogeneity of the population (i.e. males or females only, no co-morbidities, strict age group definitions, other medications, race). The advantage of the former construct is that it represents the complete target population and one could potentially identify subsets of patients who could experience side-effects or be non-responders. The disadvantage is that the heterogeneity in the two comparison groups may obscure effectiveness and further development of the intervention would be dropped.

The second option of having extensive and comprehensive exclusion criteria to make the test population RCT more homogeneous may yield statistically significant effects for the intervention, but efficacy may not extend to the real world population covering the complete spectrum of patients. Certainly, such an outcome would be a good start, but it would require more RCTs with broader patient populations and post-marketing oversight if the intervention was approved by the regulatory agency responsible.

The issue of responders and non-responders in the human population for most interventions (discussed in [36, 37]) might confound both types of RCT discussed above, since the two categories may not be evident before the assessment. Similarly, the issue of side-effects in a small number of patients may not be predictable as evidenced by the appearance of lethal complications in a small subset of patients taking a COX-2 inhibitor (i.e. Vioxx), and the genetic variability of $\mathrm{P} 450$ enzymes in humans that activate prodrugs $[13,14]$.

An additional option is the $\mathrm{N}$ of 1 construct for an experimental design where one treats each participant in a trial as an individual and studies the effect of the intervention in a "before and after" scenario to assess responsiveness vs non-responsiveness, and the pattern of responsiveness, as well as side-effects. Such a construct is not very popular with most researchers who regard the RCT as the "gold standard" based on how medicine is practiced, historical perspective, and the requirements of many regulatory agencies such as Health Canada or the FDA in the USA.

Therefore, the strength of human heterogeneity for survival of the species during evolution, and in response to recent and current threats, can be an impediment for drug or intervention acceptance to treat various diseases or conditions. However, while viewed as an impediment by many drug companies, it is very informative in its own right about human heterogeneity, the need to examine definitions of diseases and disease heterogeneity (i.e. is a tissue-specific cancer the same in all patients-melanoma, breast cancer, leukemia; is rheumatoid arthritis the same in all individuals; other chronic diseases?), and what the pattern of side-effects in individuals is actually telling the researcher/investigator.

\section{SUMMARY}

Maintaining heterogeneity in a species is a successful strategy for ensuring survival from a variety of threats originating from microbes (bacteria, viruses, fungi, parasites), food insecurity ( $>100$ genetic loci related to obesity risk [6]), bipedal mobility and specific muscle functions (running away from predators, hunting success); reproductive success as mammals (the fetus as an allograft); and developing symbiotic relationships with microbes (gut, lung skin, vagina microbiomes), particularly when no real effective interventions are available. Thus, heterogeneity should be embraced and understood in its context. With the advent of artificial intelligence (AI) and machine learning (ML) developments, and their application to the interpretation of such heterogeneity, it is now possible to start to elucidate some critical contributors from the complexity presented by human heterogeneity [38-40].

However, heterogeneity is the bane of many research efforts because it has the potential to cloud the clarity of answers to questions that are posed. Thus, many research efforts focus on eliminating as much heterogeneity as possible in order to obtain clear answers and develop clear conclusions. This perspective 
is encouraged by many granting agencies who want the funds invested to yield clear answers. Unfortunately, clear answers may be nice, but irrelevant to the population as a whole. This applies to studies of humans, as well as rodents (one may get a clear answer using an inbred mouse, but if one uses five inbred strains and gets five clear answers, how can that be interpreted regarding translation to humans?).

Perhaps, one should step back and inquire as to how to further embrace heterogeneity, examine what the complexity is providing with more introspection, and ask if the gold standards are providing the framework that is necessary to better understand the heterogeneity. Thus, with improved AI and ML tools, and new approaches to address complexity (i.e. systems analysis), it may be time to readjust our thinking with regard to the purpose of much research and how it is manifested.

\section{ACKNOWLEDGEMENTS}

The author thanks a number of colleagues and collaborators over the past several decades for the many delightful and provocative discussions on the topics addressed. The preparation of this manuscript was supported by funds from the AHS Strategic Clinical Network Program.

\section{REFERENCES}

1. Rubini, M., Gualdi-Russo, E., Manson, V.S., Rinaldo, N. and Bianucci, R. (2016) Mortality Risk Factors Show Similar Trends in Modern and Historic Populations Exposed to Plague. The Journal of Infection in Developing Countries, 10, 488-493. https://doi.org/10.3855/jidc.7974

2. Almadhi, M.A., Abdulrahman, A., Alawadhi, A., Rabaan, A.A., Atkin, S. and Al Qahtani, M. (2021) The Effect of ABO Blood Group and Antibody Class on the Risk of COVID-19 Infection and Severity of Clinical Outcomes. Scientific Reports, 11, Article No. 5745. https://doi.org/10.1038/s41598-021-84810-9

3. Telis, N., Aguilar, R. and Harris, K. (2020) Selection against Archaic Hominin Genetic Variation in Regulatory Regions. Nature Ecology \& Evolution, 4, 1558-1566. https://doi.org/10.1038/s41559-020-01284-0

4. Greer, C., Bhakta, H., Ghanem, L., Refai, F., Linn, E. and Avelia, M. (2021) Deleterious Variants in Genes Regulating Mammalian Reproduction in Neanderthals, Denisovans and Extant Humans. Human Reproduction, 36, 734-755. https://doi.org/10.1093/humrep/deaa347

5. Zeberg, H. and Paabo, S. (2020) The Major Genetic Risk Factor for Severe COVID-19 Is Inherited from Neanderthals. Nature, 587, 610-612. https://doi.org/10.1038/s41586-020-2818-3

6. Froguel, P. (2015) Obesity Genes and Their Effect on the Energy Balance. Bulletin de L'Académie Nationale de Médecine, 199, 1269-1279. (In French) https://doi.org/10.1016/S0001-4079(19)30812-X

7. Misra, M.K., Damotte, V. and Hollenbach, J.A. (2018) The Immunogenetics of Neurological Disease. Immunology, 153, 399-414. https://doi.org/10.1111/imm.12869

8. Mozzi, A., Pontremoli, C. and Sironi, M. (2018) Genetic Susceptibility to Infectious Diseases: Current Status and Future Perspectives from Genome-Wide Approaches. Infection, Genetics and Evolution, 66, 286-307. https://doi.org/10.1016/j.meegid.2017.09.028

9. Shapiro, M.R., Thirawatananond, P., Peters, L., Sharp, R.C., Ogundare, S., Posgai, A.L., Perry, D.J. and Brusko, T.M. (2021) De-Coding Genetic Risk Variants in Type 1 Diabetes. Immunology and Cell Biology. Online Ahead of Print. https://doi.org/10.1111/imcb.12438

10. Beer, A.E., Quebbeman, J.F., Ayers, J.W. and Haines, R.F. (1981) Major Histocompatibility Complex Antigens, Maternal and Paternal Immune Responses, and Chronic Habitual Abortions in Humans. The American Journal of Obstetrics and Gynecology, 141, 987-999. https://doi.org/10.1016/S0002-9378(16)32690-4

11. Billingham, R.E. and Beer, A.E. (1984) Reproductive Immunology: Past, Present and Future. Perspectives in Biology and Medicine, 27, 259-275. https://doi.org/10.1353/pbm.1984.0042

12. Ober, C., Steck, T., van der Ven, K., Billstrand, C., Messer, L., Kwak, J., Bearman, K. and Beer, A. (1993) MHC 
Class II Compatibility in Aborted Fetuses and Term Infants of Couples with Recurrent Spontaneous Abortion. Journal of Reproductive Immunology, 25, 195-207. https://doi.org/10.1016/0165-0378(93)90063-N

13. Guengerich, F.P. (2020) Cytochrome P450 2E1 and Its Roles in Disease. Chemico-Biological Interactions, 322, Article ID: 109056. https://doi.org/10.1016/j.cbi.2020.109056

14. Elewa, H. and Wilby, K.J. (2017) A Review of Pharmacogenetics of Antimalarials and Associated Clinical Implications. European Journal of Drug Metabolism and Pharmacokinetics, 42, 745-756. https://doi.org/10.1007/s13318-016-0399-1

15. El Rouby, S., Mestres, C.A., LaDuca, F.M. and Zucker, M.L. (2004) Racial and Ethnic Differences in Warfarin Response. The Journal of Heart Valve Disease, 13, 15-21.

16. Kokras, N., Dalla, C. and Papadopoulou-Daifoti, Z. (2011) Sex Differences in Pharmacokinetics of Antidepressants. Expert Opinion on Drug Metabolism \& Toxicology, 7, 213-226. https://doi.org/10.1517/17425255.2011.544250

17. Cataldi, M., Muscogiuri, G., Savastano, S., Barrea, L., Guida, B., Taglialatela, M. and Salao, A. (2019) Gender-Related Issues in the Pharmacology of New Anti-Obesity Drugs. Obesity Research, 20, 375-384. https://doi.org/10.1111/obr.12805

18. Hart, D.A. (2018) Are We Learning as Much as Possible from Space Flight to Better Understand Health and Risks to Health on Earth? Journal of Biomedical Science and Engineering, 11, 109-118. https://doi.org/10.4236/jbise.2018.116010

19. Hart, D.A. (2019) Influence of Space Environments on System Physiologic and Molecular Integrity: Redefining the Concept of Human Health beyond the Boundary Conditions of Earth. Journal of Biomedical Science and Engineering, 12, 400-408. https://doi.org/10.4236/jbise.2019.128031

20. Ray, E.A. (1991) Introduction: Are Aging and Space Effects Similar? Experimental Gerontology, 26, 123-129. https://doi.org/10.1016/0531-5565(91)90002-4

21. Vernikos, J. and Schneider, V.S. (2010) Space, Gravity, and the Pathophysiology of Aging-Parallel or Convergent Disciplines: A Mini Review. Gerontology, 56, 157-166. https://doi.org/10.1159/000252852

22. Liesegang, A., Risteli, J. and Wanner, M. (2006) The Effects of First Gestation and Lactation on Bone Metabolism in Dairy Goats and Milk Sheep. Bone, 38, 794-802. https://doi.org/10.1016/j.bone.2005.11.006

23. Liu, X., On, R.T.H., Pillai, E.N., Elzein, A.M., Small, K.S., Clark, T.G., Kwiatkowski, D.P. and Teo, Y.Y. (2013) Detecting and Characterizing Genomic Signatures of Positive Selection in Global Populations. The American Journal of Human Genetics, 92, 866-881. https://doi.org/10.1016/j.ajhg.2013.04.021

24. Roche, B., Rougeron, V., Quintana-Murci, L., Renaud, F., Abbate, J.L. and Prugnolle, F. (2017) Might Interspecies Interactions between Pathogens Drive Host Evolution? The Case of Plasmodium Species and Duffy-Negativity in Human Populations. Trends in Parasitology, 33, 21-29. https://doi.org/10.1016/j.pt.2016.09.010

25. Brown, C.A., Pappoe-Ashong, P.J., Duah, N., Ghansah, A., Asmah, H., Afari, E. and Koram, K.A. (2021) High Frequency of the Duffy-Negative Genotype and Absence of Plasmodium vivax Infections in Ghana. Malaria Journal, 20, 99. https://doi.org/10.1186/s12936-021-03618-0

26. Missaggia, B.O., Reales, G., Cybis, G.B., Hunemeir, T. and Bortolini, M.C. (2020) Adaptation and Co-Adaptation of Skin Pigmentation and Vitamin D Genes in Native Americans. American Journal of Medical Genetics. Part C, Seminars in Medical Genetics, 184, 1060-1077. https://doi.org/10.1002/ajmg.c.31873

27. Lin T.K., Man, M.Q., Abuabara, K., Wakefield, J.S., Sheu, H.M., Tsai, J.C., Lee, C.H. and Elias, P.M. (2019) By Protecting against Cutaneous Inflammation, Epidermal Pigmentation Provided an Additional Advantage for Ancestral Humans. Evolutionary Applications, 12, 1960-1970. https://doi.org/10.1111/eva.12858

28. Hart, D.A. (2016) Sex-Specific Effects of LiCl Treatment on Preservation of Renal Function and Extended Li- 
fespan in Murine Models of SLE: Perspectives on Insights into the Potential Basis for Survivorship in NZB/NZW Female Mice. Biology of Sex Differences, 7, 31. https://doi.org/10.1186/s13293-016-0085-7

29. Haddadeen, C., Lai, C., Cho, S.Y. and Healy, E. (2015) Variants of the Melanocortin-1 Receptor: Do They Matter Clinically? Experimental Dermatology, 24, 5-9. https://doi.org/10.1111/exd.12540

30. Tian, H., Lyu, Y., Yang, Y.G. and Hu, Z. (2020) Humanized Rodent Models for Cancer Research. Frontiers in Oncology, 10, 1696. https://doi.org/10.3389/fonc.2020.01696

31. Laudanski, K. (2021) Humanized Mice as a Tool to Study Sepsis-More than Meets the Eye. International Journal of Molecular Sciences, 22, 2403. https://doi.org/10.3390/ijms22052403

32. Barton, K.I., Shekaforoush, M., Heard, B.J., Vakil, P., Atarod, M., Martin, C.R., Achari, Y., Hart, D.A., Frank, C.B. and Shrive, N.G. (2017) Use of Preclinical Surgically Induced Models to Understand Biomechanical and Biological Consequences of PTOA Development. Journal of Orthopaedic Research, 35, 454-465. https://doi.org/10.1002/jor.23322

33. Gallant-Behm, C.L., Olson, M.E. and Hart, D.A. (2006) Cytokine and Growth Factor mRNA Expression Patterns Associated with the Hypercontracted, Hyperpigmented Healing Phenotype of Red Duroc Pigs: A Model of Abnormal Human Scar Development. Journal of Cutaneous Medicine and Surgery, 9, 165-177. https://doi.org/10.1177/120347540500900403

34. Vakiel, P., Shekarforoush, M., Dennison, C.R., Scott, M., Frank, C.B., Hart, D.A. and Shrive, N.G. (2020) Stress Measurements on the Articular Cartilage Surface Using Fiber Optic Technology and In-Vivo Gait Kinematics. Annals of Biomedical Engineering, 48, 2836-2845. https://doi.org/10.1007/s10439-020-02516-x

35. Vakiel, P., Shekarforoush, M., Dennison, C.R., Achari, Y., Muench, G., Scott, M., Hart, D.A. and Shrive, N.G. (2020) Correlation of Damage Score in PTOA with Changes in Stress on Cartilage in an Ovine Model. Osteoarthritis Cartilage Open, 2, Article ID: 100109. https://doi.org/10.1016/j.ocarto.2020.100109

36. Kydd, A.S.R. and Hart, D.A. (2020) Efficacy and Safety of Platelet-Rich Plasma Injections for Osteoarthritis. Current Treatment Options in Rheumatology, 6, 87-98. https://doi.org/10.1007/s40674-020-00142-1

37. Pham, T., et al. (2004) OMERACT-OARSI Initiative: Osteoarthritis Research Society International Set of Responder Criteria for Osteoarthritis Clinical Trials Revisited. Osteoarthritis Cartilage, 12, 89-99. https://doi.org/10.1016/j.joca.2004.02.001

38. Metri, R., Mohan, A., Nsengimana, J., Pozniak, J., Molina-Paris, C., Newton-Bishop, J., et al. (2017) Identification of a Gene Signature for Discriminating Metastatic from Primary Melanoma Using a Molecular Interaction Network Approach. Scientific Reports, 7, Article ID: 17314. https://doi.org/10.1038/s41598-017-17330-0

39. Zhao, J., Feng, Q.P., Wu, P., Warner, J.L., Denny, J. and Wei, W.Q. (2019) Using Topic Modeling via Non-Negative Matrix Factorization to Identify Relationships between Fenetic Variants and Disease Phenotypes: A Case Study of Liporotein (a) (LPA) PLoS ONE, 14, e0212112. https://doi.org/10.1371/journal.pone.0212112

40. Squillario, M., Abate, G., Tomasi, F., Tozzo V., Barla, A., Yberti, D., et al. (2020) A Telescope GWAS Analysis Strategy, Based on SNPs-Genes-Pathways Ensamble and on Multivariate Algorithms, to Characterize Late Onset Alzheimer's Disease. Scientific Reports, 10, Article No. 12063. https://doi.org/10.1038/s41598-020-67699-8 